Pacific Journal of Mathematics

RINGS ALL OF WHOSE FINITELY GENERATED MODULES 


\title{
RINGS ALL OF WHOSE FINITELY GENERATED MODULES ARE INJECTIVE
}

\author{
B. L. OSOFSKY
}

The main purpose of this paper is to prove that a ring all of whose finitely generated modules are injective must be semi-simple Artin. ${ }^{1}$

We begin with the following information about the class of rings under consideration:

LEMMA 1. Let $R$ be a ring with identity, and assume each cyclic right $R$-module is injective. Then $R$ is regular in the sense of von Neumann and $R$ is right self injective.

Proof. For any ring $R$ with identity, it is easy to see that a right ideal $I$ of $R$ is generated by an idempotent if and only if $I$ is a direct summand of the right $R$-module $R_{R}$. If $I$ is an injective right ideal of $R$, then $I$ is a direct summand of $R_{R}$, and therefore is generated by an idempotent. Thus if every cyclic right $R$-module is injective, each principal right ideal $a R$ generated by $a \in R$ is generated by an idempotent, that is $a R=e R$ for some $e=e^{2} \in R$. Then there exist $x, y \in R$ such that $e=a x$, and $a=e y$. It follows that $e a=e(e y)=$ $e y=a$ and $a=e a=a x a$. Thus $R$ is a regular ring, and since $R_{R}$ is generated by the identity, $R_{R}$ is injective.

Let $M_{R}$ denote a right module over a ring $R$. If $P, N$ are submodules of $M$, let $P^{\prime} \supseteqq N$ signify that $P$ is an essential extension of $N$. (See Eckmann and Schopf [2].) Then $N$ is an essential submodule of $P$.

For each $x \in M$, let $x^{R}=\{r \in R \mid x r=0\}$. The singular submodule $Z(M)$ is defined by:

$$
Z(M)=\left\{x \in M \mid R_{R}{ }^{\prime} \supseteqq x^{R}\right\}
$$

$Z\left(R_{R}\right)$ is actually a two sided ideal of $R$.

If $e=e^{2} \in R$, and if $x \in e^{R} \cap e R$, then $x=e x=0$ and so $e^{R} \cap e R=0$. Thus $Z\left(R_{R}\right)$ contains no idempotents $\neq 0$. In particular, if $R$ is a

Received July 18, 1963. This result forms part of the author's doctoral dissertation written at Rutgers University under the direction of Professor Carl Faith. The author gratefully acknowledges partial support from the National Science Foundation under grant G-19863.

1 After the author obtained this characterization of rings whose cyclic modules are injective, a translation of a recent paper [5] by L. A. Skornjakov was published and brought to the author's attention. Although Skornjakov states the major portion of the author's main theorem, his proof is incorrect. In the proof of his Lemma 9, which is crucial to his proof of the theorem, Skornjakov assumes that the injective hull of a submodule in an injective module must be a unique submodule, whereas in general it is unique only up to isomorphism.

Added in proof April 13, 1964. This lemma is actually false. See the author's dissertation for a counter-example. 
regular ring, $Z\left(R_{R}\right)=0$. (Cf. R. E. Johnson [4]).

We need the following important (and known [3]) lemma:

Lemma 2. Let $M_{R}$ be a module such that $Z(M)=0$. Then each submodule $N$ of $M$ has a unique maximal essential extension $N^{*}$ in $M$.

Proof. Let $\left\{M_{i} \mid i \in I\right\}$ be the set of all submodules of $M$ such that $M_{i}{ }^{\prime} \supseteqq N$. Set $N^{*}=\sum_{i \in I} M_{i}$. Then if $N^{*}{ }^{\prime} \supseteqq N, N^{*}$ must be the unique maximal essential extension of $N$ in $M$ since it contains every essential extension of $N$ in $M$.

For each $y \in M$, let

$$
(N: y)=\{r \in R \mid y r \in N\} \text {. }
$$

If $M \supseteqq Q^{\prime} \supseteqq N$, then $R_{R}{ }^{\prime} \supseteqq(N: y)$ for all $y \in Q$. (This follows, since any non-zero right ideal $I$ of $R$ which satisfies $I \cap(N: y)=0$, also satisfies $y I \neq 0$ and $y I \cap N=0$, a contradiction.)

Now let $0 \neq x=x_{i_{1}}+\cdots+x_{i_{n}}, 0 \neq x_{i_{j}} \in M_{i_{j}}, j=1, \cdots, n$, be any element of $N^{*}$. Then

$$
(N: x) \supseteqq \bigcap_{j=1}^{n}\left(N: x_{i_{j}}\right) .
$$

Now $M_{i_{j}}{ }^{\prime} \supseteqq N$, so $R_{R}{ }^{\prime} \supseteqq\left(N: x_{i_{j}}\right)$, and therefore $R_{R}{ }^{\prime} \supseteqq \bigcap_{j=1}^{n}\left(N: x_{i_{j}}\right)$, hence $R_{R}{ }^{\prime} \supseteqq(N: x)$. Since $Z(M)=0, x(N: x) \neq 0$, and so $x(N: x)$ is a nonzero submodule of $x R \cap N$. This proves $N^{* \prime} \supseteqq N$ as asserted.

We next consider certain properties of idempotents in a right self injective regular ring. Let $N \sim A$ denote the set theoretic complement of a set $A$ in a set $N$.

Lemma 3. Let $\left\{e_{n} \mid n \in N\right\}$ be a set of orthogonal idempotents in a right self injective regular ring. Then for every subset $A$ of $N$, there exists an idempotent $E_{A} \in R$ such that

$$
\begin{array}{lr}
E_{A} e_{n}=e_{n} & \text { for all } n \in A \\
e_{n} E_{A}=E_{A} e_{n^{\prime}}=0 & \text { for } \text { all } n^{\prime} \in N \sim A \\
E_{A}+E_{N \sim A}=E_{N} . &
\end{array}
$$

Proof. Since $R$ is regular, $Z\left(R_{R}\right)=0$. Then, by Lemma 2, each right ideal $I$ of $R$ has a unique maximal essential extension $I^{*}$ in $R$. Since $R_{R}$ is injective, by [2] $I$ has an injective hull in $R_{R}$ which is a maximal essential extension of $I$ in $R_{R}$. Thus each right ideal $I$ has a unique injective hull $I^{*}$ in $R_{R}$. Then as remarked in the proof of Lemma 1, there exists $e=e^{2} \in R$ such that $I^{*}=e R$.

Hence for any subset $A$ of $N$, there exists an idempotent $e_{A} \in R$ such that 


$$
e_{A} R=\left(\sum_{n \in A} e_{n} R\right)^{*}
$$

Since $\left\{e_{n} \mid n \in N\right\}$ are orthogonal,

$$
\left(\sum_{n \in A} e_{n} R\right) \cap\left(\sum_{n^{\prime} \in N \sim A} e_{n^{\prime}} R\right)=0 .
$$

Then $e_{A} R \cap e_{N \sim A} R=0$ (for $x \neq 0 \in e_{A} R \cap e_{N \sim A} R$ implies $x R \cap \sum_{n \in A} e_{n} R \cap$ $\sum_{n^{\prime} \in N \sim A} e_{n^{\prime}} R \neq 0$, a contradiction.) Thus the sum $e_{A} A+e_{N \sim A} R$ is direct, and since each summand is injective, $e_{A} R \oplus e_{N \sim A} R$ is injective. Since injective hulls of right ideals of $R$ are unique,

$$
\begin{aligned}
& \left(\sum_{n \in N} e_{n} R\right)^{*} \supseteqq\left(\sum_{n \in A} e_{n} R\right)^{*}, \\
& \left(\sum_{n \in N} e_{n} R\right)^{*} \supseteqq\left(\sum_{n^{\prime} \in N \sim A} e_{n^{\prime}} R\right)^{*}
\end{aligned}
$$

so $\left(\sum_{n \in N_{N}} e_{n} R\right)^{*} \supseteqq e_{A} R \bigoplus e_{N \sim A} R \supseteqq \sum_{n \in N} e_{n} R$. Then it follows $\left(\sum_{n \in N} e_{n} R\right)^{*}=$ $e_{A} R \oplus e_{N \sim A} R$. Set $E_{N}=e_{N}$, where $e_{N} R=\left(\sum_{n \in N} e_{n} R\right)^{*}$. Let $E_{A}$ (respectively $E_{N \sim A}$ ) be the projection of the idempotent $E_{N}$ on $e_{A} R$ (respectively $e_{N \sim A} R$ ). We note that $E_{A}+E_{N \sim A}=E_{N}$ by definition, and $E_{A}$ is simply the projection of the identity element of $R$ on $e_{A} R$ with respect to the direct decomposition $R=\left(1-E_{N}\right) R \oplus e_{A} R \oplus e_{N \sim A} R$. Thus $E_{A}$ and $E_{N \sim A}$ are orthogonal. Furthermore

$$
\begin{array}{lr}
E_{A} e_{n}=e_{n} & \forall n \in A \\
E_{A} e_{n^{\prime}}=E_{A} E_{N \sim A} e_{n^{\prime}}=0 & \forall n^{\prime} \in N \sim A .
\end{array}
$$

Since $e_{A} R^{\prime} \supseteqq \sum_{n \in A} e_{n} R,\left(\sum_{n \in A} e_{n} R: E_{A}\right)$ is an essential right ideal of R. But

$$
e_{n^{\prime}} E_{A}\left(\sum_{n \in A} e_{n} R: E_{A}\right) \leqq e_{n^{\prime}}\left(\sum_{n \in A} e_{n} R\right)=0 \quad \forall n^{\prime} \in N \sim A .
$$

Thus $e_{n^{\prime}} E_{A} \in Z\left(R_{R}\right)=0$, and we conclude $e_{n^{\prime}} E_{A}=0 \forall n^{\prime} \in N \sim A$.

LEMMA 4. Let $R$ be a right self injective regular ring which contains an infinite set of orthogonal idempotents $\left\{e_{n} \mid n \in N\right\}$. Let $I=\sum \oplus e_{n} R$. For $A \subseteq N$, let $E_{A}$ be defined as in Lemma 3. Then a set $S_{\mathfrak{A}}=\left\{E_{A} \mid A \in \mathfrak{A}\right\}$, where each $A$ is infinite, is independent modulo $I$, that is, $\sum_{A \in \mathfrak{Q}}\left(E_{A} R+I\right)$ is direct in $R-I$, if and only if for any finite set $\left\{A_{i} \mid i=1, \cdots, n\right\} \subseteq \mathfrak{A}, A_{i} \cap \bigcup_{j \neq i} A_{j}$ is a finite subset of $N, 1 \leqq i \leqq n$.

Proof. Assume $S_{\mathscr{A}}$ is independent modulo $I$, and let $\left\{A_{i} \mid i=\right.$ $1, \cdots, n\} \cong \mathfrak{A}$. Set $C=C_{i j}=A_{i} \cap A_{j}$. For all $i$ and $j \neq i, E_{A_{i}} R \supseteqq$ $\sum_{n \in O} e_{n} R$ and $E_{A_{j}} R \supseteqq \sum_{n \in O} e_{n} R$. Thus $E_{A_{i}} R \supseteqq\left(\sum_{n \in O} e_{n} R\right)^{*}=E_{o} R$ and 
$E_{A_{j}} R \supseteqq\left(\sum_{n \in O} e_{n} R\right)^{*}=E_{o} R$. Since $0=E_{C}-E_{o}=E_{A_{i}} E_{\sigma}-E_{A_{j}} E_{\sigma}, E_{C} \in I$. Then for all but a finite number of $n \in C, e_{n} E_{\sigma}=0$. Since this implies $e_{n}=e_{n} E_{0} e_{n}=0$ which is true for no $n, C$ must be finite. Then $A_{i} \cap \mathrm{U}_{j \neq i} A_{j}$ is a finite union of finite sets, and thus finite.

Now assume $A_{i} \cap \bigcup_{j \neq i} A_{j}$ is finite, and let $\sum_{j=1}^{n} E_{A_{j}} r_{j} \in I$. If $m \notin A_{i}$, $e_{m} E_{A_{i}} r_{i}=0$ by Lemma 3. If $m \in A_{i} \sim \bigcup_{j \neq i} A_{j}, e_{m} \sum_{j=1}^{n} E_{A_{j}} r_{j}=e_{m} E_{A_{i}} r_{i}$. Since $\sum_{j=1}^{n} E_{A_{j}} r_{j} \in I$, there are at most a finite number of $m \in A_{i} \sim \mathrm{U}_{j \neq i} A_{j}$ such that $e_{m} E_{A_{i}} r_{i} \neq 0$. Since $A_{i} \cap \bigcup_{j \neq i} A_{j}$ is also finite, the set

$$
B=\left\{m \in N \mid e_{m} E_{A_{i}} r_{i} \neq 0\right\}
$$

must be finite.

Now for all $n^{\prime} \in N \sim B$,

$$
\begin{aligned}
0 & =e_{n^{\prime}} E_{A_{i}} r_{i}=e_{n^{\prime}}\left(\left[1-E_{N}\right]+E_{N \sim B}+E_{B}\right) E_{A_{i}} r_{i} \\
& =e_{n^{\prime}} E_{N \sim B} E_{A_{i}} r_{i} .
\end{aligned}
$$

Assume $E_{N \sim B} E_{A_{i}} r_{i} \neq 0$. Then, since $E_{N \sim B} R^{\prime} \supseteqq \sum_{n^{\prime} \in N \sim B} e_{n^{\prime}} R$, there is an $s \in R$ such that $E_{N \sim B} E_{A_{i}} r_{i} s \neq 0 \in \sum_{n^{\prime} \in N \sim B} e_{n^{\prime}} R$, so for some $n^{\prime} \in N \sim B$, $e_{n}, E_{N \sim B} E_{A_{i}} r_{i} s \neq 0$, a contradiction.

Then

$$
E_{A_{i}} r_{i}=\left(\left[1-E_{N}\right]+E_{N \sim B}+E_{B}\right) E_{A_{i}} r_{i}=E_{B} E_{A_{i}} r_{i} .
$$

Since a finite direct sum of injective modules is injective, $\sum_{i \in_{B}} e_{i} R=$ $\left(\sum_{i \in B} e_{i} R\right)^{*}=E_{B} R$ and $E_{B} R \cong I$. It follows that $E_{A_{i}} r_{i} \in I$.

LEMMA 5. Let $R$ be a right self injective regular ring which contains an infinite set of orthogonal idempotents $\left\{e_{n} \mid n \in N\right\}$. If $I=\sum_{n \in N} e_{n} R$, then $R-I$ is not an injective $R$-module.

Proof. Let $\left\{A_{i} \mid i=1,2, \cdots\right\}$ be a countable family of subsets $A_{i} \leqq N$ such that $\left\{E_{A_{i}} \mid i=1,2, \cdots\right\}$ are independent in $R-I$. For example, the $A_{i}$ may be disjoint countable subsets of $N$.

Let $\mathscr{P}$ denote the family of sets $S_{\mathfrak{A}}=\left\{E_{B_{\alpha}} \mid B_{a} \leqq N, \alpha \in \mathfrak{X}\right\}$ where $\mathfrak{A}$ is some index set, such that $S_{\mathfrak{X}} \supseteqq\left\{E_{A_{i}} \mid i=1,2, \cdots\right\}$ and $S_{\mathfrak{X}}$ is independent modulo $I$. Partially order $\mathscr{P}$ by inclusion. Since independence modulo $I$ depends only on finite sets of idempotents, $\mathscr{P}$ is inductive. By Zorn's lemma, select a maximal element $S \in \mathscr{P}$.

Let $J=\sum_{E_{B} \in S} E_{B} R$. Define $\varphi: J \rightarrow R-I$ by

$$
\begin{array}{rlrl}
\varphi\left(E_{A_{i}}\right) & =E_{A_{i}}+I & \forall i=1,2, \cdots \\
\varphi\left(E_{B}\right) & =0+I & \forall E_{B} \in S \sim\left\{E_{A_{i}}\right\} \\
\varphi\left(\sum_{k=1}^{n} E_{B_{k}} r_{k}\right) & =\sum_{k=1}^{n} \varphi\left(E_{B_{k}}\right) r_{k} & & E_{B_{k}} \in S, r_{k} \in R .
\end{array}
$$

$\sum_{k=1}^{n} E_{B_{k}} r_{k}=0$ implies $E_{B_{k}} r_{k} \in I$ since the idempotents of $S$ are independent 
modulo $I$. Hence $\varphi\left(E_{B_{k}}\right) r_{k}=0+I$, and $\varphi\left(\sum_{k=1}^{n} E_{B_{k}} r_{k}\right)=0+I$. Thus $\varphi$ is a map which is clearly an $R$ homomorphism.

Assume $\varphi$ is induced by left multiplication by $m+I$ in $R-I$, $m \in R$. Then

$$
m E_{A_{i}}-E_{A_{i}} \in I \quad \forall i=1,2, \cdots
$$

and

$$
m E_{B} \in I \quad \forall E_{B} \in S \sim\left\{E_{A_{i}}\right\} .
$$

From (1) we conclude that for all but a finite number of $n \in A_{i}$, $e_{n}\left(m E_{A_{i}}-E_{A_{i}}\right)=0$ and $e_{n} m E_{A_{i}} e_{n}=e_{n} E_{A_{i}} e_{n}$. Thus $e_{n} m e_{n}=e_{n}$ by Lemma 3.

From (2) we conclude that for all but a finite number of $n^{\prime} \in B$, $e_{n^{\prime}} m E_{B}=0$, and $e_{n^{\prime}} m E_{B} e_{n^{\prime}}=e_{n^{\prime}} m e_{n^{\prime}}=0$.

Let $j_{1} \in A_{1}, e_{j_{1}} m e_{j_{1}}=e_{j_{1}}$. Select $j_{n+1} \in A_{n+1}$ such that

$$
e_{j_{n+1}} m e_{j_{n+1}}=e_{j_{n+1}}
$$

and

$$
j_{n+1} \notin A_{k}
$$

for all $k<n+1$.

This is possible since $\left\{j \in A_{n+1} \mid e_{j} m e_{j}=e_{j}\right\}$ is infinite and Lemma 4 implies $A_{n+1} \cap \bigcup_{k=1}^{n} A_{k}$ is finite.

Since $S$ is maximal in $\mathscr{P}$, by Lemma $4\left\{j_{n} \mid n=1,2, \cdots\right\}$ thus defined must have an infinite number of elements in common with some $B \subseteq N$ such that $E_{B} \in S . \quad B \neq A_{i} i=1,2, \cdots$ since $j_{n} \notin A_{i}$ for all $n>i$. Therefore $\varphi\left(E_{B}\right)=0$, and $e_{j^{\prime}}=e_{j^{\prime}} m e_{j^{\prime}}=0$ for all but a finite number of $j^{\prime} \in B \cap\left\{j_{n} \mid n=1,2, \cdots\right\}$. This contradicts the assumption that $B \cap\left\{j_{n} \mid n=1,2, \cdots\right\}$ is an infinite set. Thus $\varphi$ is not induced by left multiplication by $m+I$ in $R-I$. Hence $R-I$ is not injective. (See [1], p. 8.)

THEOREM. Let $R$ be a ring with 1 . Then the following conditions are equivalent:

(a) $R$ is semi-simple Artin.

(b) Every finitely generated right $R$-module is injective.

(c) Every cyclic right $R$-module is injective.

Proof. $\quad(a) \Rightarrow(b)$. By ([1], p. 11, Theorem 4.2), every right module over a semi-simple Artin ring $R$ is injective, and so every finitely generated right $R$-module is injective.

$(\mathrm{b}) \Rightarrow(\mathrm{c})$. Since every cyclic $R$-module is finitely generated by one element, (c) is a special case of (b).

(c) $\Rightarrow$ (a). If every cyclic $R$-module is injective, by Lemma $1, R$ 
is right self injective and regular. By Lemma $5, R$ cannot contain an infinite set of orthogonal idempotents. It is well known that this condition in any regular ring $R$ implies that $R$ satisfies the minimum condition and hence is semi-simple Artin.

COROLLARY. Let $R$ be a right self injective, hereditary ring with identity. Then $R$ is semi-simple Artin.

Proof. $R$ hereditary is equivalent to the condition that every quotient of an injective $R$-module is injective. (See [1], p. 14.) Since every cyclic module is isomorphic to a quotient of the injective module $R_{R}$, every cyclic $R$-module is injective. Therefore by the theorem $R$ is semi-simple Artin.

\section{REFERENCES}

1. H. Cartan and S. Eilenberg, Homological Algebra, Princeton University Press, 1956.

2. B. Eckmann and A. Schopf, Über injektive Moduln, Archiv der Mathematik, 4 (1953), 75-78.

3. Carl Faith, Theory of Rings, Lecture Notes (dittoed), Rutgers University, 1963.

4. R. E. Johnson, The extended centralizer of a ring over a module, Proc. Amer. Math. Soc., 2 (1951), 891-895.

5. L. A. Skornjakov, Rings with injective cyclic modules, Soviet Mathematics, 4 (1963), $36-38$.

Rutgers, The State University 


\title{
PACIFIC JOURNAL OF MATHEMATICS
}

\author{
EDITORS
}

\author{
Robert Osserman \\ Stanford University \\ Stanford, California \\ M. G. Arsove \\ University of Washington \\ Seattle 5, Washington
}

\author{
J. Dugundji \\ University of Southern California \\ Los Angeles 7, California \\ Lowell J. Paige \\ University of California \\ Los Angeles 24, California
}

\section{ASSOCIATE EDITORS}
E. F. BECKENBACH
B. H. NEUMANN
F. WOLF
K. YOSHIDA

\section{SUPPORTING INSTITUTIONS}

\author{
UNIVERSITY OF BRITISH COLUMBIA \\ CALIFORNIA INSTITUTE OF TECHNOLOGY \\ UNIVERSITY OF CALIFORNIA \\ MONTANA STATE UNIVERSITY \\ UNIVERSITY OF NEVADA \\ NEW MEXICO STATE UNIVERSITY \\ OREGON STATE UNIVERSITY \\ UNIVERSITY OF OREGON \\ OSAKA UNIVERSITY \\ UNIVERSITY OF SOUTHERN CALIFORNIA
}

\author{
STANFORD UNIVERSITY \\ UNIVERSITY OF TOKYO \\ UNIVERSITY OF UTAH \\ WASHINGTON STATE UNIVERSITY \\ UNIVERSITY OF WASHINGTON \\ AMERICAN MATHEMATICAL SOCIETY \\ CALIFORNIA RESEARCH CORPORATION \\ SPACE TECHNOLOGY LABORATORIES \\ NAVAL ORDNANCE TEST STATION
}

Mathematical papers intended for publication in the Pacific Journal of Mathematics should by typewritten (double spaced), and on submission, must be accompanied by a separate author's résumé. Manuscripts may be sent to any one of the four editors. All other communications to the editors should be addressed to the managing editor, L. J. Paige at the University of California, Los Angeles 24, California.

50 reprints per author of each article are furnished free of charge; additional copies may be obtained at cost in multiples of 50 .

The Pacific Journal of Mathematics is published quarterly, in March, June, September, and December. Effective with Volume 13 the price per volume (4 numbers) is $\$ 18.00$; single issues, $\$ 5.00$. Special price for current issues to individual faculty members of supporting institutions and to individual members of the American Mathematical Society: $\$ 8.00$ per volume; single issues $\$ 2.50$. Back numbers are available.

Subscriptions, orders for back numbers, and changes of address should be sent to Pacific Journal of Mathematics, 103 Highland Boulevard, Berkeley 8, California.

Printed at Kokusai Bunken Insatsusha (International Academic Printing Co., Ltd.), No. 6, 2-chome, Fujimi-cho, Chiyoda-ku, Tokyo, Japan.

PUBLISHED BY PACIFIC JOURNAL OF MATHEMATICS, A NON-PROFIT CORPORATION

The Supporting Institutions listed above contribute to the cost of publication of this Journal, but they are not owners or publishers and have no responsibility for its content or policies. 


\section{Pacific Journal of Mathematics}

\section{Vol. 14, No. $2 \quad$ June, 1964}

Tom M. (Mike) Apostol and Herbert S. Zuckerman, On the functional equation $F(m n) F((m, n))=F(m) F(n) f((m, n)) \ldots \ldots \ldots \ldots \ldots \ldots \ldots \ldots \ldots \ldots \ldots$

Reinhold Baer, Irreducible groups of automorphisms of abelian groups . . . . . . . 385

Herbert Stanley Bear, Jr., An abstract potential theory with continuous kernel . . . . 407

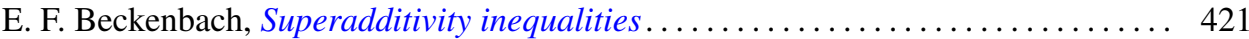

R. H. Bing, The simple connectivity of the sum of two disks . . . . . . . . . . . 439

Herbert Busemann, Length-preserving maps ...................... 457

Heron S. Collins, Characterizations of convolution semigroups of measures . . . . . . 479

Paul F. Conrad, The relationship between the radical of a lattice-ordered group and complete distributivity............................ 493

P. H. Doyle, III, A sufficient condition that an arc in $S^{n}$ be cellular . . . . . . . . . 501

Carl Clifton Faith and Yuzo Utumi, Intrinsic extensions of rings . . . . . . . . . . 505

Watson Bryan Fulks, An approximate Gauss mean value theorem . . . . . . . . . . 513

Arshag Berge Hajian, Strongly recurrent transformations . . . . . . . . . . . . . 517

Morisuke Hasumi and T. P. Srinivasan, Doubly invariant subspaces. II . . . . . . . 525

Lowell A. Hinrichs, Ivan Niven and Charles L. Vanden Eynden, Fields defined by

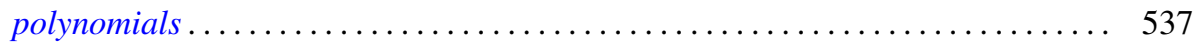

Walter Ball Laffer, I and Henry B. Mann, Decomposition of sets of group

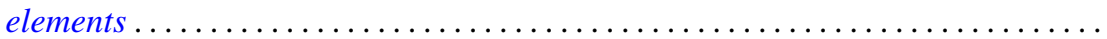

John Albert Lindberg, Jr., Algebraic extensions of commutative Banach

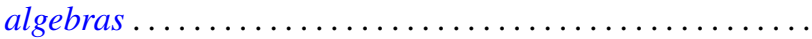

W. Ljunggren, On the Diophantine equation $C x^{2}+D=y^{n} \ldots$

M. Donald MacLaren, Atomic orthocomplemented lattices ....

Moshe Marcus, Transformations of domains in the plane and applications in the

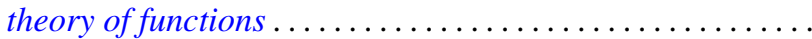

Philip Miles, $B^{*}$ algebra unit ball extremal points . .................. 627

W. F. Newns, On the difference and sum of a basic set of polynomials . . . . . . . 639

Barbara Osofsky, Rings all of whose finitely generated modules are injective ...... 645

Calvin R. Putnam, Toeplitz, matrices and invertibility of Hankel matrices . . . . . . . 651

Shoichiro Sakai, Weakly compact operators on operator algebras . . . . . . . . . 659

James E. Simpson, Nilpotency and spectral operators . . . . . . . . . . . . . 665

Walter Laws Smith, On the elementary renewal theorem for non-identically

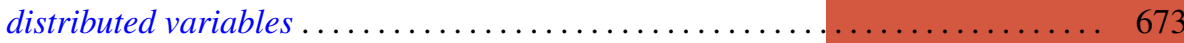

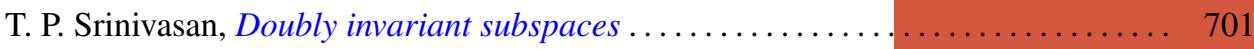

J. Roger Teller, On the extensions of lattice-ordered groups . . . . . . . . . . . . 709

Robert Charles Thompson, Unimodular group matrices with rational integers as

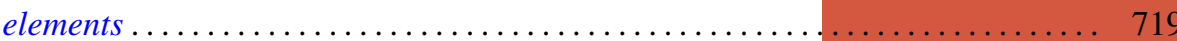

J. L. Walsh and Ambikeshwar Sharma, Least squares and interpolation in roots of unity

Charles Edward Watts, A Jordan-Hölder theorem .................... 731

Kung-Wei Yang, On some finite groups and their cohomology .............. 735

Adil Mohamed Yaqub, On the ring-logic character of certain rings ............ 741

Paul Ruel Young, A note on pseudo-creative sets and cylinders 